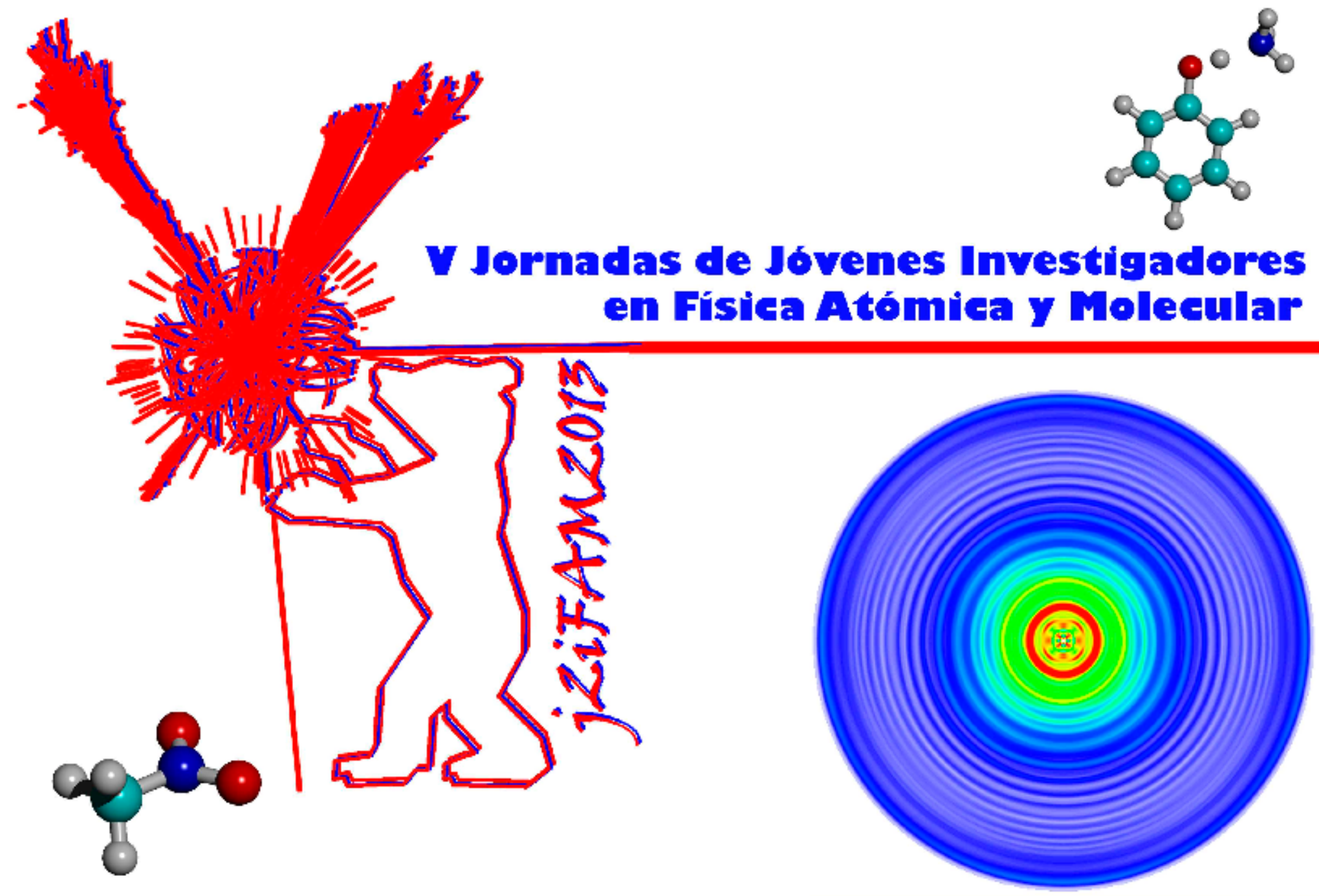

February, I 3 rd - I sth 2013 Madrid, Spain

Physies of Ageregates

Reaction Dynamics

Quantum Ghemistry

Surface Physies

Atomie and Molecular Gollisions
Ultracold Atoms and Molecules Canomaterial Seience Spectroseopy and Exeited States Quantum Information

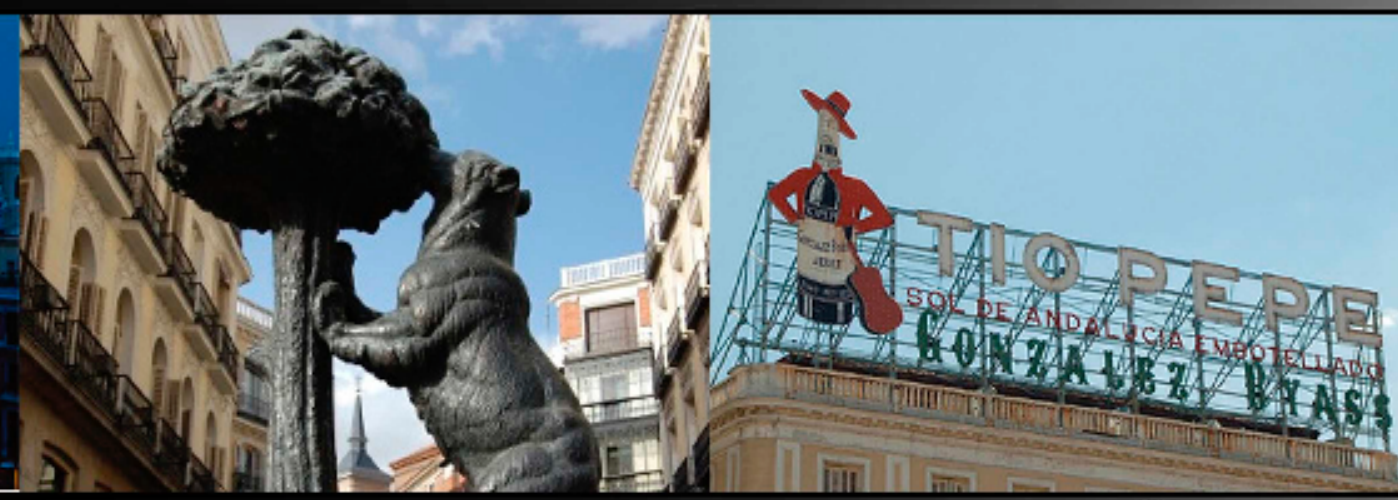

https//wrw.ucm.es/centros/webs/j2ifamv/

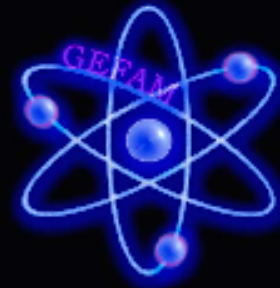

Salon de agtos

Faeultad de Gieneias Cuínietas UGM

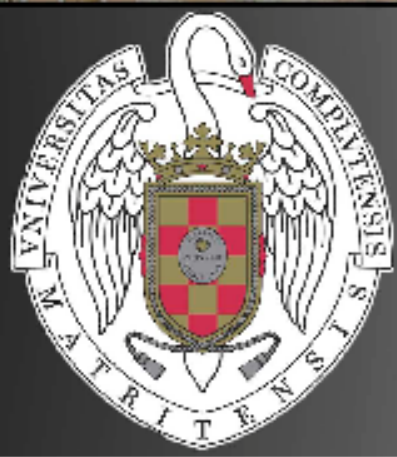




\section{Low-order harmonic generation in nanosecond laser ablation plasmas of carbon containing materials}

I. López-Quintás ${ }^{1}$, M. Oujja ${ }^{1}$, M. Sanz ${ }^{1}$, M. Martín ${ }^{1}$, R.A. Ganeev², M. Castillejo ${ }^{1}$

${ }^{1}$ Instituto de Química Física Rocasolano, CSIC, Serrano 119, 28006 Madrid, Spain

${ }^{2}$ Voronezh State University, Voronezh 394006, Russia

E-mail: ilopez@iqfr.csic.es

In order to understand and control harmonic generation ( $\mathrm{HG}$ ) processes in laser ablation plasmas [1], knowledge about the species responsible for frequency up-conversion is extremely important. HG emerges as a diagnosis tool to study the nonlinear optical properties of the emitters and in some cases to follow the spatiotemporal behaviour of the species generated in the ablation plume.

In this work, the nonlinear behaviour of laser plasmas produced from carbon containing materials (graphite and boron carbide) was investigated by ablating the targets with a nanosecond Q-switched Nd:YAG laser. The generation of low-harmonics (3rd and 5th) of the fundamental wavelength of a second nanosecond Nd:YAG driving laser, propagating perpendicularly to the ablation beam at a given temporal delay, was observed. Optical emission spectroscopy and time-of-flight-mass spectrometry measurements of the ablation plume revealed details on plasma composition and its spatiotemporal evolution. In particular, in the case of graphite targets, evidence of $\mathrm{C}_{7}$ up to $\mathrm{C}_{3} 2$ clusters was found.

In agreement with these findings, deposits collected by on-line pulsed laser deposition showed the presence of carbon based nanoaggregates. This holistic approach to ablation plume analysis allows discussing the identity of the nonlinear emitters in laser ablation plasmas and facilitates the investigation of efficient, nanoparticle-enhanced, coherent short wavelength generation processes.

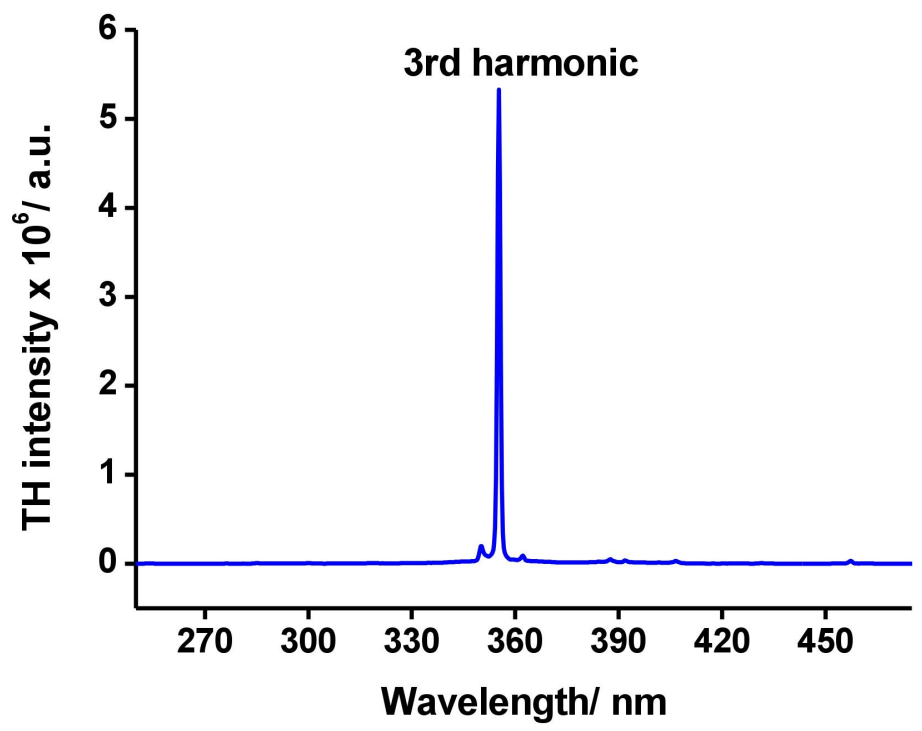

Figure 1: Third harmonic emission at $355 \mathrm{~nm}$ observed in graphite ablation plasma.

[1 ] R.A. Ganeev, J. Phys. B: At. Mol. Opt. Phys., 40, R213-R253 (2007). 